\title{
Infección por VIH, Hepatitis B y Sífilis en reclusos de centros penitenciarios de Venezuela, 1998-2001
}

\author{
Posada $\mathrm{A}^{1}$, Díaz Tremarias $\mathrm{M}^{2}$
}

${ }^{1}$ Licenciado en Bioanálisis, Coordinador de Programas y Proyectos de la Dirección Central de Extensión, Universidad Central de Venezuela.

${ }^{2}$ Magíster en Educación, Licenciado en Bioanálisis, Jefe de la Cátedra de Toxicología, Escuela de Bioanálisis, Universidad Central de Venezuela.

\section{RESUMEN}

Objetivo: Determinar la prevalencia de VIH, Hepatitis B y Sífilis en reclusos durante el período 1998-2001.

Materiales y Métodos: Estudio descriptivo, transversal realizado en 6 centros penitenciarios con una muestra de 1.773 reclusos voluntarios. Se recolectaron y analizaron muestras sanguíneas para la identificación de anticuerpos contra el VIH, anti core de la Hepatitis B y Treponema pallidum a través de inmunoensayos enzimáticos (ELISA) y RPR, respectivamente.

Resultados: Se obtuvieron 456 casos positivos de los cuales 70 (4,0\%) correspondieron a VIH, 280 (16,2\%) para Hepatitis B y 106 (6,1\%) para Sífilis. El mayor porcentaje de casos VIH positivos se ubica en los reclusos más jóvenes (18 a 37 años); el número de casos de Hepatitis B aumentó progresivamente con la edad hasta alcanzar un porcentaje máximo en el grupo de 48-57 años; no se observó diferencia estadística significativa en los casos de sífilis con relación a la edad pero si entre los casos positivos para VIH y Hepatitis B en relación con la edad, tiempo y número de centros de reclusión.

Palabras clave: Prisiones; prisioneros; sífilis; VIH; Hepatitis; Salud Pública.

\section{HIV, HEPATITIS B AND SYPHILIS INFECTION IN INMATES OF VENEZUELA'S PRISONS, 1998-2001}

\begin{abstract}
Objective: To determine the prevalence of HIV, Hepatitis B and syphilis amongst inmates during the time period 19982001.

Materials and Methods: Descriptive, cross-sectional study carried out at 6 prisons using a volunteer sample of 1773 inmates. Blood samples were gathered and analysed using enzyme linked immunoassays (ELISA) and RPR screening tests to identify the presence of HIV antibodies, Hepatitis B anti-core antibodies and treponema pallidum.

Results: 456 cases gave a positive response to tests, 70 (4,0\%) of which gave positive results for HIV, 280 (16,2\%) for Hepatitis B and 106 (6,1\%) for syphilis. The greatest percentage of HIV cases were found amongst the youngest inmates (18 to 37 years). The number of Hepatitis B cases increased progressively in line with age until reaching a maximum percentage in the 48-57 age group. No significant statistical difference for age was observed in syphilis cases, but differences were found amongst HIV positive and Hepatitis B cases for age, length of sentence and number of prisons where inmate resided.
\end{abstract}

Key words: Prisons; prisoners; syphilis; HIV; Hepatitis; Public Health.

Texto recibido: Enero 2008

Texto aceptado: Septiembre 2008 


\section{INTRODUCCIÓN}

Los conflictos tradicionales de las cárceles, la disciplina entendida como violencia, las deficiencias estructurales, el hacinamiento, la alimentación inadecuada y, los escasos servicios de salud, son fenómenos que repercuten seriamente en las condiciones de vida mínimas para sobrevivir a la pena de encierro y socava inevitablemente las relaciones intramuros. Mucho más grave resulta cuando la persona está afectada por un problema de salud, para lo cual la cárcel y las deplorables condiciones de vida en su interior, resultan fatales ${ }^{1}$.

A esto se ha agregado una problemática, que adquiere expresiones particularmente graves en este tipo de instituciones, como son las Infecciones de Transmisión Sexual (ITS) las cuales constituyen un grupo amplio pero relativamente bien definido de enfermedades infecciosas que tienen serias repercusiones sanitarias, psicológicas, sociales y económicas, por lo cual es importante su diagnóstico oportuno ${ }^{2}$.

El virus de la Inmunodeficiencia Humana (VIH), ha sido detectado en las prisiones de casi todos los países del mundo ${ }^{3,4}$ y la proporción de algunas otras infecciones también es relevante, tales como la Hepatitis B y C y la Sífilis, lo cual debería ser causa de gran preocupación, no sólo para las autoridades penitenciarias, sino para la sociedad en general, ya que existe un importante tránsito entre la prisión y la comunidad ${ }^{1}$.

Por lo tanto, el objetivo del estudio realizado, en el marco del Programa de Extensión Penitenciario de la Universidad Central de Venezuela, fue determinar la prevalencia de ITS, específicamente VIH, Hepatitis B y Sífilis, en centros penitenciarios de Venezuela durante el período 1998-2001 a fin de obtener información general de la situación de estas infecciones en la población penal, que permita diseñar intervenciones para su prevención y tratamiento con el propósito de fomentar comportamientos saludables.

Por tratarse de una población que se ha estudiado muy poco en nuestro país, desde el punto de vista epidemiológico y de laboratorio, esta investigación pudiera ser la base para la ejecución de futuros estudios en el área.

\section{MATERIALES Y MÉTODOS}

El presente es un estudio descriptivo, transversal realizado en 6 centros penitenciarios, asignados por el Ministerio del Poder Popular de Relaciones Interiores y Justicia (MPPRIJ) a criterios restringidos de interés para la institución, durante el periodo comprendido entre los años 1998-2001. De la población total de internos masculinos en esos penales al momento del estudio
( $\mathrm{N}=3348)$, se extrajeron muestras sanguíneas de 1.773 sujetos, quienes participaron de manera voluntaria, previo consentimiento informado y autorización por escrito.

Los sujetos fueron entrevistados a través de un cuestionario o ficha epidemiológica, elaborado a tal fin, en referencia a sus datos personales, condición jurídica (nacionalidad, número de centros y tiempo de reclusión) y posibles conductas de riesgo para la adquisición de ITS.

Las muestras sanguíneas, una vez centrifugadas, fueron trasladadas al servicio de laboratorio encargado de su procesamiento y conservadas a $-70^{\circ} \mathrm{C}$ hasta el momento de su análisis.

Para la detección de infección sifilítica se utilizó una prueba de tamizaje de Reagina Plasmática Rápida (RPR) de Vue Benton Dickinson y para la determinación de anticuerpos VIH, tipos 1 y 2, y anticuerpo anti core Hepatitis B se utilizaron los respectivos métodos de Inmunoensayo Enzimático cualitativo (ELISA) de Ortho Diagnostic Systems.

El análisis estadístico de la información suministrada por los internos en el cuestionario y los resultados de la muestras de sangre se llevó a cabo haciendo uso de medidas descriptivas tales como frecuencia, porcentaje, media, medianas y la desviación típica, con el fin de describir el grupo de estudio según los valores obtenidos; y también a través de medidas de asociación, como Chi-cuadrado y comparación de promedios mediante el estadístico z. Para ello se utilizó el programa Excel 2000 de Microsoft Office bajo ambiente Windows y el SPSS v 9.0.

Los resultados de las pruebas de laboratorio aplicadas fueron entregados en formato individual a cada uno de los internos participantes y el informe respectivo se consignó ante la División de Medicina Integral del Ministerio del Interior y Justicia, y en el Servicio Médico de cada centro penitenciario.

\section{RESULTADOS}

De las 1.773 muestras evaluadas se obtuvieron 456 casos positivos para las ITS investigadas, lo que representa un $26,3 \%$ de estas infecciones en la población estudiada. De éstas, 70 casos $(4,0 \%)$ correspondieron a internos que presentaron anticuerpos contra el VIH, $280(16,2 \%)$ y $106(6,1 \%)$ presentaron anticuerpos contra el core de Hepatitis B y anticuerpos antitreponémicos, respectivamente.

En la Tabla I se detallan los resultados obtenidos en cada centro penitenciario del estudio, pudiendo observar que el Centro Penitenciario Centro Occidental fue el que presentó mayor porcentaje $(34,6 \%)$ de internos con alguna de las ITS investigadas, seguido del Inter- 
nado Judicial Yaracuy con $29,8 \%$ y el Centro Penitenciario Región Capital con 27,3\% de casos positivos.

También observamos que es en el C.P. Región Capital donde se detecta el mayor número de casos positivos para VIH (6\%); en el C. P. Centro Occidental y en el I. J. Yaracuy se registran el mayor número de casos positivos para Hepatitis B (28,8\%) y sífilis $(10,6 \%)$, respectivamente.

Con relación a la edad, el 51,7\% de la población evaluada se encontraba entre los 18 y 27 años de edad. En la Tabla II se presenta la prevalencia de las ITS en estudio según los grupos etarios de los sujetos participantes, observando que el mayor porcentaje de casos VIH positivos se ubica en los estratos más jóvenes, es decir, entre 18 a 37 años; en contraposición con el número de casos de Hepatitis B que aumentó progresivamente con la edad hasta alcanzar un porcentaje máximo en el grupo de 48-57 años; existiendo diferencia estadística significativa en los casos de VIH y Hepatitis B con respecto a la edad, no así en cuanto a la Sífilis.

En la Tabla III se muestra el porcentaje de ITS con relación al tiempo de reclusión de la población evalua- da, evidenciándose, que el mayor porcentaje de casos de VIH $(6,2 \%)$ y Hepatitis B $(33 \%)$ lo encontramos en aquellos internos que tenían cinco años de reclusión. Se observó diferencia estadísticamente significativa entre los casos positivos para Hepatitis B y el tiempo de reclusión. En relación con la sífilis, los mayores porcentajes de casos positivos ( $8,9 \%$ y $8,3 \%$ ) se encontraron en los internos que tenían dos y tres años de reclusión, respectivamente.

La Tabla IV indica la prevalencia de las ITS estudiadas en relación con el número de centros en los cuales han estado recluidos los sujetos que participaron en la investigación, observando que a medida que aumenta el número de estos centros también aumenta el porcentaje de positividad de estas infecciones. En el caso del VIH, el mayor número de casos positivos $(5,9 \%)$ fueron detectados en los internos que han estado recluidos en al menos tres establecimientos. Para la $\mathrm{He}-$ patitis $\mathrm{B}$, observamos un porcentaje creciente de casos positivos a medida que aumenta el número de centros en los que han estado recluidos los internos que resultaron positivos a esta entidad.

\begin{tabular}{lccccccccc}
\hline & \multicolumn{7}{c}{ Infecciones de Transmisión Sexual } & \multicolumn{2}{c}{} \\
Centros Penitenciarios & Total & VIH & \% & Hep B & \% & Sífilis & \% & Total ITS & \% \\
\hline Total & 1733 & 70 & 4,0 & 280 & $\mathbf{1 6 , 2}$ & 106 & $\mathbf{6 , 1}$ & 456 & $\mathbf{2 6 , 3}$ \\
I. J. Falcón & 121 & 2 & 1,7 & 8 & $\mathbf{6 , 6}$ & 6 & $\mathbf{5 , 0}$ & 16 & $\mathbf{1 3 , 2}$ \\
C. P. Centro Occidental & 191 & 0 & 0,0 & 55 & $\mathbf{2 8 , 8}$ & 11 & $\mathbf{5 , 8}$ & 66 & $\mathbf{3 4 , 6}$ \\
P. G. Venezuela & 173 & 3 & $\mathbf{1 , 7}$ & 36 & $\mathbf{2 0 , 8}$ & 5 & $\mathbf{2 , 9}$ & 44 & $\mathbf{2 5 , 4}$ \\
I. J. Yaracuy & 114 & 4 & $\mathbf{3 , 5}$ & 18 & $\mathbf{1 5 , 8}$ & 12 & $\mathbf{1 0 , 5}$ & 34 & $\mathbf{2 9 , 8}$ \\
I. J. Monagas & 135 & 1 & $\mathbf{0 , 7}$ & 19 & $\mathbf{1 4 , 1}$ & 3 & $\mathbf{2 , 2}$ & 23 & $\mathbf{1 7 , 0}$ \\
C. P. Región Capital & 999 & 60 & $\mathbf{6 , 0}$ & 144 & $\mathbf{1 4 , 4}$ & $\mathbf{6 9}$ & $\mathbf{6 , 9}$ & 273 & $\mathbf{2 7 , 3}$ \\
\hline
\end{tabular}

I.J.: Internado Judicial; C.P.: Centro Penitenciario; P.G.: Penitenciaria General

Tabla I. Infecciones de Transmisión Sexual en internos evaluados por centro de reclusión en Venezuela para el período 1998-2001.

\begin{tabular}{|c|c|c|c|c|c|c|c|}
\hline \multirow[b]{2}{*}{ ITS } & \multicolumn{7}{|c|}{ Grupos de edad } \\
\hline & Total & $18-27$ & $28-37$ & $38-47$ & $48-57$ & $58-67$ & \\
\hline & Total & 1733 & 896 & 564 & 202 & 57 & 14 \\
\hline \multirow{4}{*}{$\mathrm{VIH}^{1}$} & Positivo & 70 & 37 & 25 & 6 & 2 & 0 \\
\hline & $\%$ & 4,0 & 4,1 & 4,4 & 3,0 & 3,5 & 0,0 \\
\hline & Negativo & 1663 & 859 & 539 & 196 & 55 & 14 \\
\hline & $\%$ & 96,0 & 95,9 & 95,6 & 97,0 & 96,5 & 100,0 \\
\hline \multirow[t]{4}{*}{ Hepatitis $\mathrm{B}^{1}$} & Positivo & 280 & 139 & 91 & 35 & 13 & 2 \\
\hline & $\%$ & 16,2 & 15,5 & 16,1 & 17,3 & 22,8 & 14,3 \\
\hline & Negativo & 1453 & 757 & 473 & 167 & 44 & 12 \\
\hline & $\%$ & 83,8 & 84,5 & 83,9 & 82,7 & 77,2 & 85,7 \\
\hline \multirow[t]{4}{*}{ Sífilis } & Positivo & 106 & 56 & 33 & 13 & 4 & 0 \\
\hline & $\%$ & 6,1 & 6,3 & 5,9 & 6,4 & 7,0 & 0,0 \\
\hline & Negativo & 1627 & 840 & 531 & 189 & 53 & 14 \\
\hline & $\%$ & 93,9 & 93,8 & 94,1 & 93,6 & 93,0 & 100,0 \\
\hline
\end{tabular}

${ }^{1}$ Diferencias significativas entre las edades de los reclusos con VIH y Hepatitis B. $(\mathrm{z}=1,64 ; \mathrm{p}<0,05)$

Tabla II. Prevalencia de ITS en internos evaluados según grupo etario, Venezuela. 1998-2001. 


\begin{tabular}{|c|c|c|c|c|c|c|c|c|c|}
\hline \multirow[b]{2}{*}{ ITS } & & \multicolumn{8}{|c|}{ Tiempo de reclusión (años) } \\
\hline & & Total & 0 & 1 & 2 & 3 & 4 & 5 & 6 ó más \\
\hline & Total & $1732^{*}$ & 639 & 189 & 237 & 230 & 169 & 97 & 171 \\
\hline \multirow[t]{4}{*}{$\mathrm{VIH}$} & Positivo & 70 & 18 & 5 & 13 & 10 & 10 & 6 & 8 \\
\hline & $\%$ & 4,0 & 2,8 & 2,7 & 5,5 & 4,4 & 5,9 & 6,2 & 4,7 \\
\hline & Negativo & 1662 & 621 & 184 & 224 & 220 & 159 & 91 & 163 \\
\hline & $\%$ & 96,0 & 97,2 & 97,3 & 94,5 & 95,6 & 94,1 & 93,8 & 95,32 \\
\hline \multirow[t]{4}{*}{ Hepatitis $\mathrm{B}^{1}$} & Positivo & 280 & 34 & 30 & 44 & 49 & 49 & 32 & 42 \\
\hline & $\%$ & 16,2 & 5,3 & 15,9 & 18,6 & 21,3 & 28,9 & 33 & 24,6 \\
\hline & Negativo & 1452 & 605 & 159 & 193 & 181 & 120 & 65 & 129 \\
\hline & $\%$ & 83,8 & 94,7 & 84,1 & 81,4 & 78,7 & 71,0 & 67,0 & 75,4 \\
\hline \multirow[t]{4}{*}{ Sífilis } & Positivo & 106 & 30 & 12 & 21 & 19 & 8 & 3 & 13 \\
\hline & $\%$ & 6,1 & 4,7 & 6,4 & 8,9 & 8,3 & 4,7 & 3,1 & 7,6 \\
\hline & Negativo & 1626 & 609 & 177 & 216 & 211 & 161 & 94 & 158 \\
\hline & $\%$ & 93,9 & 95,3 & 93,6 & 91,1 & 91,7 & 95,2 & 96,9 & 92,4 \\
\hline
\end{tabular}

* Un interno no respondió.

${ }^{1}$ Diferencias significativas según los tiempos de reclusión: $x=110,6 ; p=0,01$

Tabla III. Prevalencia de ITS en internos evaluados con relación al tiempo de reclusión, Venezuela. 1998-2001.

\begin{tabular}{|c|c|c|c|c|c|c|c|}
\hline \multirow{3}{*}{ ITS } & \multicolumn{7}{|c|}{ Número de Centros en los que han estado recluidos } \\
\hline & & Total & 1 & 2 & 3 & 4 & 5 ó más \\
\hline & Total & 1733 & 608 & 780 & 219 & 75 & 51 \\
\hline \multirow[t]{4}{*}{$\overline{\mathrm{VIH}^{1}}$} & Positivo & 70 & 14 & 38 & 13 & 4 & 1 \\
\hline & $\%$ & 4,04 & 2,30 & 4,87 & 5,94 & 5,33 & 1,96 \\
\hline & Negativo & 1663 & 594 & 742 & 206 & 71 & 50 \\
\hline & $\%$ & 95,96 & 97,70 & 95,13 & 94,06 & 94,67 & 98,04 \\
\hline \multirow[t]{4}{*}{ Hepatitis $\mathrm{B}^{2}$} & Positivo & 280 & 63 & 133 & 44 & 22 & 18 \\
\hline & $\%$ & 16,16 & 10,36 & 17,05 & 20,09 & 29,33 & 35,29 \\
\hline & Negativo & 1453 & 545 & 647 & 175 & 53 & 33 \\
\hline & $\%$ & 83,84 & 89,64 & 82,95 & 79,91 & 70,67 & 64,71 \\
\hline \multirow[t]{4}{*}{ Sífilis } & Positivo & 106 & 25 & 58 & 9 & 8 & 6 \\
\hline & $\%$ & 6,12 & 4,11 & 7,44 & 4,11 & 10,67 & 11,76 \\
\hline & Negativo & 1627 & 583 & 722 & 210 & 67 & 45 \\
\hline & $\%$ & 93,88 & 95,89 & 92,56 & 95,89 & 89,33 & 88,24 \\
\hline
\end{tabular}

${ }^{1}$ Diferencias significativas según números de centros de reclusión: $x=9,05 ; p<0,05$

${ }^{2}$ Diferencias significativas según números de centros de reclusión: $x=41,4 ; p<0,05$

Tabla IV. Prevalencia de ITS en internos evaluados con relación al número de centros en los cuales han estado recluidos, Venezuela. $1998-2001$.

\section{DISCUSIÓN}

Para el año 1999 se registraron 21.233 casos de ITS a nivel nacional ${ }^{5}$, sin embargo no fue posible confrontar los datos obtenidos, en nuestro estudio, con los oficiales, en virtud de no existir información específica al respecto dado que, los casos de ITS de los centros penitenciarios son incluidos en la información general de cada entidad federal. Vale la pena mencionar que en nuestra investigación se detectaron 60 casos en 1998 en un solo penal y 456 casos positivos en los seis penales investigados para el período 1998-2001. Algo más de la cuarta parte de la población estudiada presentó al menos una de las ITS investigadas y aunque pareciera un porcentaje no tan elevado, si lo comparamos con la población general, consideramos que es preocupante, debido a que la persona afectada por una de estas infecciones, posee unas deplorables condiciones de vida, una pobre atención de salud y un importante tránsito hacia otros penales y con la comunidad en general. 
Con relación al VIH, mientras cifras oficiales indican 7.500 casos en el país entre 1997 y $1998^{5}$ y el ente oficial encargado de estos datos, reconoce un subregistro de hasta $75 \%$ de los $\operatorname{casos}^{6}$, organismos internacionales estiman en más de 85.000 el número de personas que viven con VIH/Sida en Venezuela para ese período ${ }^{7}$. En los datos acumulados en el país durante el período 1996-2000 se registran 228 casos de VIH en el Distrito Capital y nosotros encontramos 60 casos en 1998 en solo uno de los penales ubicados en esa zona geográfica. Este hallazgo es preocupante, pues dadas las características de este contexto, se garantiza la progresión de la enfermedad, además de su transmisión y asociación con otras infecciones como la tuberculosis y la hepatitis C.

En el año 2001 se registran 6.000 nuevos casos positivos de VIH a nivel nacional, estimándose un subregistro del $90 \%$. Para esa misma fecha, se refieren un total de 18 casos de VIH en las cárceles del país, reconociendo continuar sin un registro confiable sobre el número de reclusos seropositivos y con SIDA en los centros de reclusión. El ente oficial responsable de estos datos atribuye el subregistro a fallas en el sistema de vigilancia epidemiológica, prejuicios y discriminación; negación de la epidemia como un problema de salud pública y desconocimiento por parte del personal de salud ${ }^{8}$. En España se estima que un $9 \%$ de los casos SIDA en prisión ha eludido la detección?.

La situación de los casos VIH en reclusos de países latinoamericanos también arroja datos alarmantes. En Guatemala la detección de VIH en las prisiones se inició en 1988, con un resultado de siete reclusos portadores; en 1989 se detectaron otros cuatro casos y en 1990 doce, entre los meses de marzo y abril, para la población de reclusos que, a la fecha, oscilaba entre 6.000 y $7.000^{10}$. Existe un $6,78 \%$ de seropositividad en la población reclusa en Argentina, con un nivel más alto entre jóvenes de 18-21 años, aunque estudios más recientes ha elevado la cifra hasta un $22 \%$; hasta octubre de 1997 se reportó un acumulado de $180 \mathrm{ca}$ sos de SIDA, sólo en las prisiones de la capital mexicana; mientras que en Costa Rica, en los últimos cuatro años, se han diagnosticado 110 casos de VIH/SIDA dentro del sistema penitenciario ${ }^{11}$. En un estudio realizado en reclusos de una cárcel de Brasil, se reportó una prevalencia de $16 \%, 34 \%$ y $18 \%$ para HIV, Hepatitis C y sífilis, respectivamente ${ }^{12}$.

La prevalencia del VIH en las cárceles de Francia, es 10 veces mayor que en la población general, mientras que en EEUU existían 5,2 casos de SIDA por cada 1.000 reclusos en 1994, casi el séxtuplo que en la población general ${ }^{7}$. En investigación realizada en 450 reclusos de una cárcel de Andalucía, el 19,1\% dijo ser seropositivo para HIV, dato similar al obtenido en el estudio de todas las personas $(n=1.663)$ que ingresaron en el Centro Penitenciario de León entre enero 1991 y diciembre $1995(19,4 \%)^{3}$ y en el ámbito nacional español $(19,9 \%)^{1}$.

También para la Hepatitis B se evidencia un alto subregistro en nuestro país, ya que los datos oficiales nos reportan que entre 1996-2000 se han detectado un promedio de 975 casos y en nuestra investigación, encontramos 280 casos en el período 1998-2001 únicamente en las seis instituciones penitenciarias evaluadas. En relación con los casos de sífilis identificados $(6,1 \%)$, no se disponen de datos específicos nacionales ni a nivel penitenciario, que permitan comparar nuestros hallazgos, sin embargo obtuvimos cifras similares a las observadas en mujeres trabajadoras del sexo comercial en México ${ }^{13}$.

Si bien es probable que algunos de los internos evaluados que resultaron positivos ya se encontraran infectados desde antes de estar presos, muchos provienen de sectores de la población con un riesgo mayor de contraer estas infecciones en comparación con el resto de la sociedad. Es importante resaltar que los casos positivos de Hepatitis B fueron significativamente mayores en edad, tiempo de reclusión y número de centros en los que han estado recluidos con respecto a los casos que resultaron negativos, lo cual no sólo es contraproducente para la salud integral del interno y su familia, el control y seguimiento del caso sino que desde el punto de vista epidemiológico conlleva a un alto riesgo de transmitir esta enfermedad y contraer otras ${ }^{1,3,14,15,16}$. Adicionalmente, tal como es fácil suponer, en nuestras cárceles persisten conductas de riesgo que fomentan la presencia y mantenimiento de estas enfermedades, conductas que serán presentadas y discutidas en detalle en entrega posterior.

Con respecto a la situación del VIH en el país, los datos oficiales señalan que entre 1982 y 2006 se han registrado 63.000 casos, sin embargo organismos internacionales estiman entre 200.000 a 400.000 personas infectadas; en este sentido, Venezuela es considerada el país con mayor subregistro de la región andina; los grupos etarios más afectados son los adultos entre 25 y 30 años (60\%); la principal vía de transmisión es la sexual (57\%) en tanto que la transmisión por uso de drogas endovenosas resulta un problema menor para el país ${ }^{17}$.

Para septiembre 2007, no se pudo contar con información oficial sobre el estado de salud general de la población penitenciaria, tampoco el diagnóstico institucional realizado durante el año 2005 (en el mar- 
co de un convenio suscrito entre Cuba y Venezuela) arrojó mayor información al respecto, cuando se reconoció el desconocimiento sobre el estado de salud de la población, que no existía clasificación por patologías ni programas de salud dirigidos a la prevención y control de enfermedades transmisibles y crónicas. Sin embargo, para esa misma fecha se registró que el $0,8 \%$ de la población reclusa se encontraba infectada por $\mathrm{VIH}^{18}$, sin olvidar que las cifras mantienen un subregistro histórico importante.

No quisiéramos concluir sin mencionar que este trabajo tiene no solo las limitaciones propias de los diseños transversales sino que adicionalmente no fue posible practicar ensayos analíticos confirmatorios, por razones logísticas y económicas; no obstante consideramos que el estudio realizado constituye un antecedente importante en el contexto penitenciario pues aporta información valiosa al investigador interesado en el tema y conforma una base referencial de datos.

El negar o hacer caso omiso de los datos presentados no ayudará a resolver el problema de la existencia y propagación de ITS en centros penitenciarios venezolanos, por lo tanto aspiramos a que los mismos se conviertan en una llamada de alerta y estímulo para sumar disposiciones y voluntades, a fin de garantizar acciones contundentes, progresivas y eficaces para el logro de la prevención, diagnóstico y tratamiento de éstas y otras afecciones, pues sería imposible revertir la situación de salud en los establecimientos penitenciarios sin una política de salud sostenida y sin atender y mejorar las precarias condiciones de reclusión en nuestro país.

\section{CORRESPONDENCIA}

Universidad Central de Venezuela,

Facultad de Medicina, Escuela de Bioanálisis,

Piso 3, Cátedra de Toxicología,

Caracas 1051, Venezuela

Dirección correo electrónico:

mariseladt@hotmail.com; angelsuc@cantv.net

\section{REFERENCIAS BIBLIOGRÁFICAS}

1. Ramos M, Oviedo-Joekes E, March JC, Prieto MA, Ballesta R. Infección de HIV, hepatitis C y tuberculosis autoreferida en una cárcel de Andalucía, 1999. Rev Esp Sanid Pent. 2006; 8 (1): 10-18.
2. Posada, A. Historia General de las Instituciones Penitenciarias. En/In Posada A, Salazar E. Las Cárceles... una visión. Caracas. Ediciones del Rectorado de la Universidad Central de Venezuela. 2004: 13-36.

3. Martin V, Caylá JA, Moris ML, Alonso LE, Pérez R. Evolución de la prevalencia de infección por virus de la Inmunodeficiencia Humana en población reclusa al ingreso en prisión durante el período 1991-1995. Rev Esp Salud Pública 1997; 71 (3): 269-280.

4. Consejo Editorial. Las prisiones; una nueva oportunidad para la Salud. (Editorial). Rev Esp Sanid Penit 2005; 7 (1): 1-3.

5. Ministerio de Salud y Desarrollo Social. Dirección de Epidemiología y Análisis Estratégico. Venezuela, 1999.

6. Programa Venezolano de Educación-Acción en Derechos Humanos. Informe Anual 1997-1998. Derecho a la salud. Caracas, Autor. [Fecha de acceso 15 de diciembre de 2002] Disponible en http:// www.derechos.org.ve

7. Organización Mundial de la Salud OMS-ONUSIDA Resumen Mundial sobre la Pandemia VIH/SIDA. Informe del grupo de trabajo ONUSIDA OMS sobre la vigilancia mundial del VIH/SIDA y las ETS, 1998.

8. Ministerio del Poder Popular para Relaciones Interiores y Justicia. Dirección General de Custodia y Rehabilitación del Recluso. Dirección de Fiscalización e Investigación. Departamento de Estadísticas, Venezuela, 2001.

9. Acín E, Gómez P, Hernando P, Corella I. Incidencia de casos SIDA en Instituciones Penitenciarias. Método de captura-recaptura. Año 2000. Rev Esp Sanid Penit 2003; 5 (1): 3-7

10. Arango, JL. Prisión y Sida. Testimonio de una Experiencia Profesional. Políticas de Salud Pública y SIDA. Guatemala: Anius, 1997.

11. SIDALAC. SIDA y drogadicción agrava crisis en cárceles de América Latina (marzo 2000). [Fecha de acceso 4 de abril de: 2000]. Disponible en www.unaids.org

12. Buratitini MN, Massad E, Rozman M, Azevedo RS, Carvalho HB. Correlation between HIV and $\mathrm{HCV}$ in Brazilian prisoners: evidence for parenteral transmission inside prison. Rev. Saúde Pública 2000; 34 (5): 431-436.

13. Hernández Girón C, Cruz Valdez A, Juárez L, Hernández Ávila M. Prevalencia y factores de riesgo asociados a sífilis en mujeres. Rev Saüde Pública 1998; 32 (6): 579-586. 
14. Wasserheit, JN. Epidemiological Synergy. Interrelationships between Human Immunodeficiency Virus Infection and Other Sexually Transmitted Diseases. Sexually Transmitted Diseases 1992; 19: 61-77.

15. Arazo Garcés P. Una propuesta de manejo práctico del paciente coinfectado en el medio penitenciario. Rev. Esp Sanid Penit 2006; 8 (3): 88-94

16. Saíz de la Hoya P, Bedia M, Esteban PJ, Figueira JJ, Guerrero JA, de Juan J et al. Características y situación clínica de la población reclusa infectada por el VIH de los centros penitenciarios españoles. Rev Esp Sanid Penit 2006; 8 (1): 19-27.
17. Programa Venezolano de Educación-Acción en Derechos Humanos. Informe Anual 2006-2007. Derecho a la salud. Caracas, Autor. [Fecha de acceso 10 de diciembre de 2007]. Disponible en http://www.derechos.org.ve

Programa Venezolano de Educación-Acción en Derechos Humanos. Informe anual 2006-2007. Derechos de las personas detenidas y encarceladas. Caracas, Autor. [Fecha de acceso 10 de diciembre de 2007]. URL Disponible en http:// www.derechos.org.ve 